\title{
Bond Paths and van der Waals Interactions in Orpiment, $\mathrm{As}_{2} \mathrm{~S}_{3}$
}

\author{
G. V. Gibbs, ${ }^{*} \dagger$ A. F. Wallace, ${ }^{\ddagger}$ R. Zallen, ${ }^{\S}$ R. T. Downs, ${ }^{\|}$N. L. Ross, ${ }^{\perp}$ D. F. Cox, ${ }^{\#}$ and \\ K. M. $\operatorname{Rosso}^{\nabla}$ \\ Departments of Geosciences, Materials Science and Engineering and Mathematics, Department of Physics, \\ Department of Chemical Engineering, Virginia Tech, Blacksburg, Virginia 24061, U.S.A., Earth Science \\ Division, Lawrence Berkeley National Laboratory, Berkeley, California 94720, Department of Geosciences, \\ University of Arizona, Tucson, Arizona 85721, U.S.A., and Chemical and Materials Science Division, and the \\ W.R. Wiley Environmental Molecular Sciences Laboratory, Pacific Northwest National Laboratory, Richland, \\ Washington 99352, U.S.A
}

Received: March 16, 2010; Revised Manuscript Received: April 27, 2010

\begin{abstract}
The calculated electron density distribution for orpiment, $\mathrm{As}_{2} \mathrm{~S}_{3}$, reveals that $\mathrm{As}-\mathrm{S}, \mathrm{S}-\mathrm{S}$, and $\mathrm{As}-\mathrm{As}$ bond paths are associated with the experimental interlayer directed bonded interactions detected in a combined infrared and Raman study. The successful modeling of the infrared- and Raman-determined interlayer bonded interactions together with bond paths and the structuralization of a variety of inorganic molecules in terms of "key-lock" bond path mainstays support the argument that van der Waals forces in inorganic molecular crystals are directional.
\end{abstract}

\section{Introduction}

Orpiment, $\mathrm{As}_{2} \mathrm{~S}_{3}$, is a 2:3 coordinated material that has been studied extensively by solid-state experimentalists. It acts as a large-bandgap semiconductor at ambient conditions and absorbs light in the blue, imparting to the mineral its diagnostic yelloworange color. ${ }^{1}$ High-pressure experiments show that the bandgap decreases rapidly with increasing pressure, ${ }^{2}$ resulting in a progressive change in color from yellow-orange to red to black. ${ }^{3}$ The effect of pressure is to shorten the weak interlayer van der Waals, vdW, bonded interactions, resulting in an increase in the interlayer splittings and the widths of both the valence and conduction bands accompanied with the reduction of the bandgap. $^{2}$ The optical properties of the mineral have also been studied in the far-infrared region in terms of the lattice vibrations and the interlayer interactions. ${ }^{4}$

The orpiment crystal structure consists of corrugated layers of heart-shaped six-membered rings of corner sharing $\mathrm{AsS}_{3}$ pyramids (Figure 1a). As the layers are neutral and extend indefinitely in all directions parallel to (010), the individual layers are considered to be macromolecular layers of $\mathrm{As}_{2} \mathrm{~S}_{3}$ composition held together by weak vdW dipole-induced nondirectional intermolecular forces. ${ }^{5-7}$ However, the nature of the forces that structuralize the layers as a three-dimensional (3D) crystal is poorly understood, although the assumption of nondirectional vdW forces is often made in the absence of better information.

In a comprehensive review of a number of experimentally determined molecular crystals, Kitaigorodskii ${ }^{8}$ concluded that the molecules in organic molecular crystals tend to fill space as efficiently as possible, adopting a cheek-by-jowl close-packed

\footnotetext{
* To whom correspondence should be addressed.

† Departments of Geosciences, Materials Science and Engineering and Mathematics, Virginia Tech.

* Lawrence Berkeley National Laboratory

$\S$ Department of Physics, Virginia Tech.

"University of Arizona.

${ }^{\perp}$ Department of Geosciences, Virginia Tech.

\# Department of Chemical Engineering, Virginia Tech.

$\nabla$ Pacific Northwest National Laboratory.
}

(a)

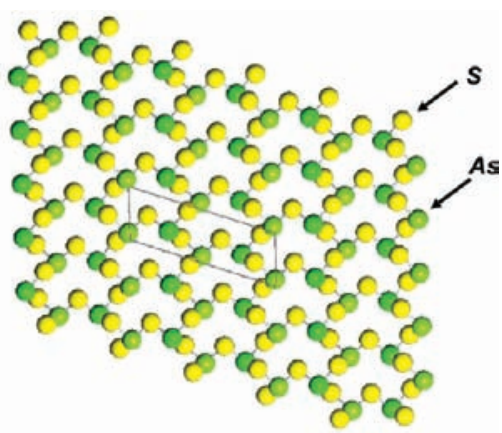

(b)

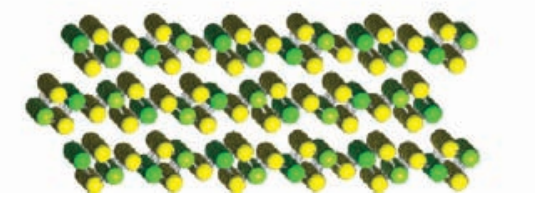

Figure 1. Drawings of an $\mathrm{As}_{2} \mathrm{~S}_{3}$ layer for orpiment viewed (a) parallel to $\mathbf{b}^{*}$ and (b) three macromolecular layers viewed roughly parallel to a. The coordinates of the atoms used to make the drawing were transformed to the $P 2_{1} / c$ setting from the $P 2_{1} / n$ setting determined by Mullen and Nowacki ${ }^{6}$ (see Table 1 for the transformed cell dimensions and atomic coordinates). The yellow spheres represent the sulfur atoms and the green ones represent arsenic atoms. The intramolecular bonded interactions that link the arsenic and sulfur atoms are colored silver.

arrangement, as may be expected for a structure governed by nondirectional, nonlocalized intermolecular induced-dipole vdW forces. ${ }^{9,10}$ On the other hand, he concluded that directional forces play a dominate role in the structuralization of the molecules in inorganic crystals, indicating that forces other than those associated with induced-dipole nondirectional interactions may be at play.

As observed by Morimoto, ${ }^{5}$ the macromolecular layers in orpiment contain distinctive spiral chains of AsS composition running parallel to [100]. These chains are viewed end-on in the rectangular pattern of $\mathrm{AsS}_{3}$ pyramids in Figure 1b. The corrugated layers are stacked in a cheek-by-jowl fashion with 
TABLE 1: Bond Paths for Orpiment ${ }^{6}$

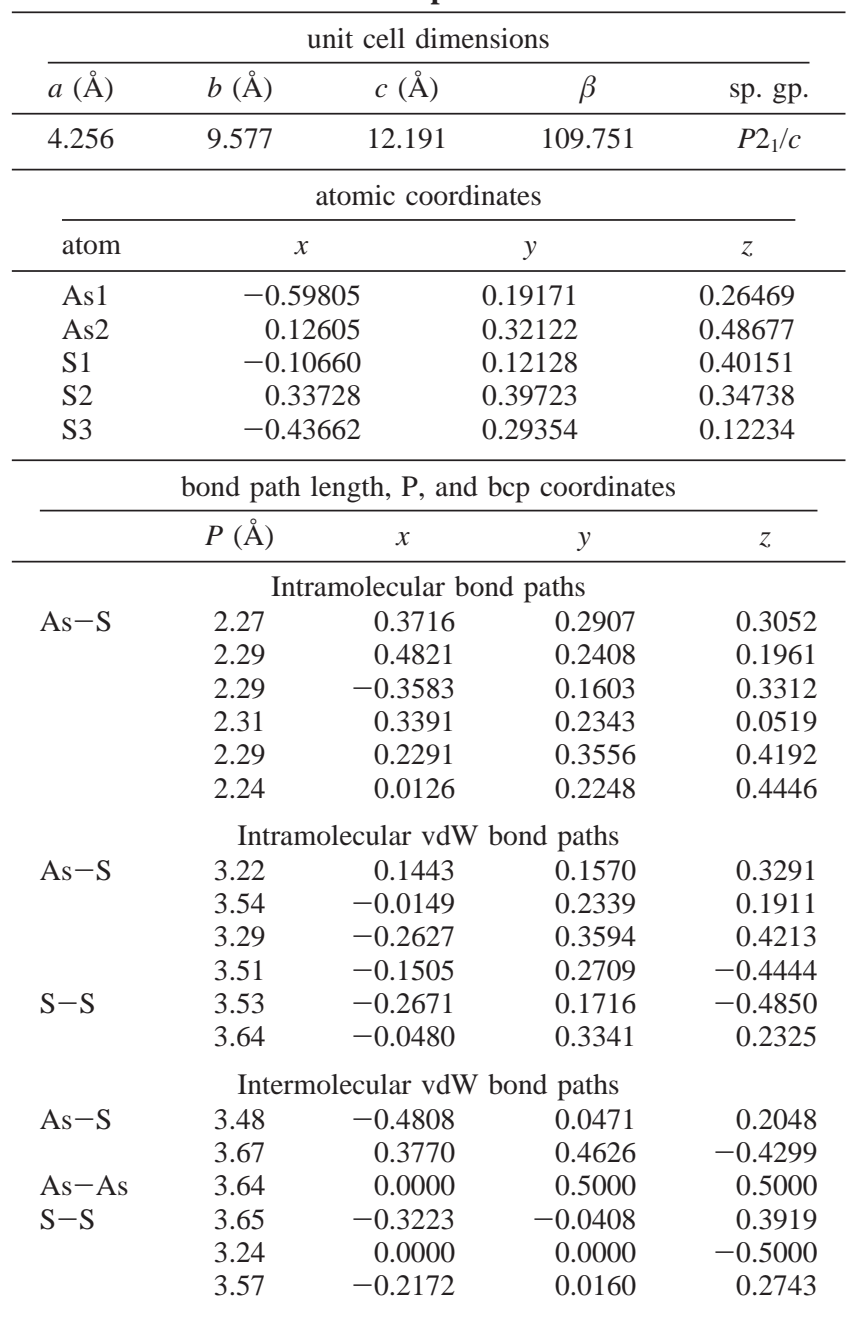

the thickness maxima (spiral chains) and minima (interchain sulfur bridges) of the layers mutually nesting together in an echelon fashion. As shown in a detailed analysis of the splittings of the vibrational bands for 2:3 coordinated materials, DeFonzo and Tauc ${ }^{11}$ established that the spiral chains play a fundamental role among the intralayer (internal mode) vibrations for materials like orpiment. A study of the distinct splittings detected in the low frequency rigid-layer vibrational modes of the Raman and infrared spectra revealed the presence of directed interlayer $S-S$ bonded interactions between the macromolecular layers of the orpiment structure ${ }^{7}$ (Figure 2). By rigid-layer vibrational modes, we mean that the intralayer atom-atom distances remain relatively fixed while the interlayer atom-atom distances vary substantially during vibrational motion. The frequencies of the rigid layer interlayer modes indicate that the bonded interactions, ascribed to directed $\mathrm{S}-\mathrm{S}$ interactions, contribute a substantial vibrational restoring force, corresponding to a stiffness coefficient that is about $7 \%$ of the stiffness of the intralayer As $-\mathrm{S}$ shared interactions. The rigid-layer modes are believed to occur as a result of directed interlayer bonded interactions that exist between the individual macromolecular layers rather than as a result of nondirectional vdW intermolecular forces.

In an earlier study, largely ignored by the van der Waals community, Feynman ${ }^{12}$ established that $\mathrm{vdW}$ intermolecular forces arise by dint of the accumulation and the deformation of the electron density, $\mathrm{ED}$, distribution between the nuclei of a pair of bonded atoms rather than by dint of induced-dipole interactions. Using equations based on Schrödinger perturbation

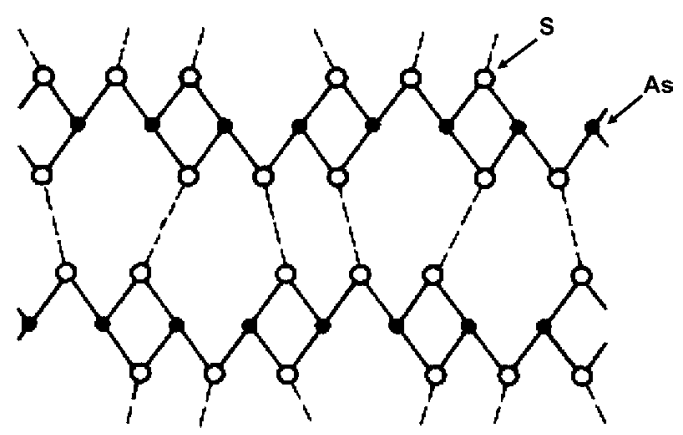

Figure 2. A highly schematic drawing of the orpiment structure viewed down a (taken from Zallen and Slade ${ }^{28}$ ). The open circles represent sulfur atoms, and the solid circles represent arsenic atoms. The intramolecular $\mathrm{As}-\mathrm{S}$ bonded interactions are represented by the solid lines, and the dashed lines represent the density of the interlayer van der Waals $\mathrm{S}-\mathrm{S}$ bonded interactions. The latter were used to estimate the force constants for the interlayer bonded interactions.

theory to evaluate the forces directly for a pair of bonded atoms at a separation, $R$, that is large compared with the radius sum for the pair of atoms, he not only found that the ED distribution for each of the atoms is deformed from central symmetry, but that a dipole moment is induced on each with the center of gravity of the ED distribution for the pair displaced slightly toward one another. On the basis of this result, he stated that "It is not the interaction of these dipoles which leads to van der Waals forces, but rather the attraction of each nucleus for the distorted charge distribution of its own electrons that gives the attractive $1 / R^{7}$ force." On the basis of the accumulation of the ED between the pair and the connection between the virial theorem and covalent binding, Slater ${ }^{13}$ went a step further and asserted that there is no fundamental distinction between $\mathrm{vdW}$ and shared bonded interactions. This assertion indicates that intermolecular vdW interactions are directional like shared interactions but substantially weaker. If so, one may conclude that there is a continuum of directed bonded interactions that exist between shared and vdW bonded interactions. On the basis of these conclusions, Bader ${ }^{14}$ asserted that the forces that determine the intramolecular interactions in a molecular crystal are no different from those that determine the vdW intermolecular bonded interactions that structuralize the molecules. He also concluded that the accumulation of the ED between the nuclei of a vdW bonded pair of atoms naturally results in a line of maximum ED (a bond path) linking the pair, a line that, in effect, defines the directed nature of the bonded interaction, an interaction that may be considered to play a decisive role in structuralizing the molecules in terms of the ED distribution of a molecular crystal. ${ }^{15-17}$

Studies undertaken for several inorganic molecular crystals (solid molecular chlorine, $\mathrm{Cl}_{2},{ }^{18} \mathrm{ClF},{ }^{19} \mathrm{~S}_{4} \mathrm{~N}_{4},{ }^{20}$ sénarmontite, $\mathrm{Sb}_{2} \mathrm{O}_{3},{ }^{21}$ arsenolite, $\mathrm{As}_{2} \mathrm{~S}_{3},{ }^{22}$ and $\mathrm{N}_{2} \mathrm{O}_{4}{ }^{23}$ ), have revealed that directed vdW bond paths typically connect the atoms on a given molecule where the ED is locally depleted (a Lewis acid site where the Laplacian $L(\mathbf{r})=-\nabla^{2} \rho(\mathbf{r})$ is negative) with the atoms on adjacent molecules where the ED is locally concentrated (a Lewis base site where the Laplacian $L(\mathbf{r})=-\nabla^{2} \rho(\mathbf{r})$ is positive).$^{24}$ In effect, the formation of such vdW bond paths may be considered to correspond to an intermolecular Lewis acid-base like interaction. Further, the connection between the bond paths and the Lewis acid-base domains indicates that the structuralizing forces of the molecules in an inorganic molecular crystal are encrypted in the Laplacian distribution of the valence electrons of the constituent vdW bonded atoms. In effect, the 


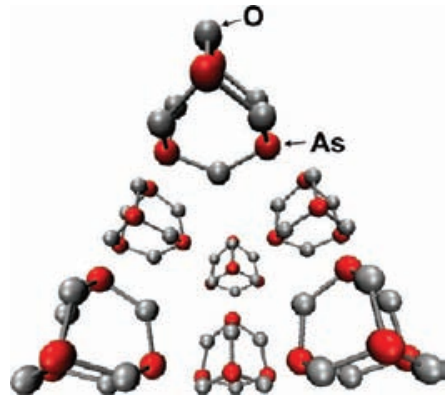

Figure 3. A perspective drawing of the structuralization of the $\mathrm{As}_{4} \mathrm{O}_{6}$ molecules in arsenolite viewed down [110]. The red spheres represent arsenic atoms, and the silver ones represent oxygen atoms. The molecules occupy one-third the space that would be occupied by a close packed array of molecules. The barycenters of the molecules are arranged like the $\mathrm{C}$ atoms in diamond with each molecule being tetrahedrally coordinated by four equivalent moleules. The end result is a molecular crystal with tetrahedral space group type symmetry $F d 3 m$ as observed for diamond.

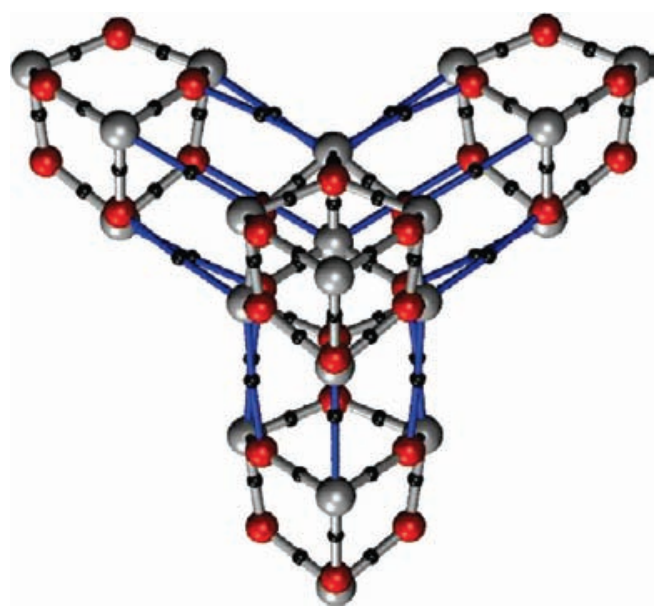

Figure 4. A picture of four equivalent $\mathrm{As}_{4} \mathrm{O}_{6}$ molecules in arsenolite connected by $\mathrm{As}-\mathrm{O}$ van der Waals bond paths. The molecules are structuralize in space by the directed $\mathrm{As}-\mathrm{O}$ bond paths such that the angles formed at the barycenter of the central molecule and that of the three adjacent molecules are $\cos ^{-1}(-1 / 3)$. The As $-\mathrm{O}$ bond paths are colored blue, and the small black spheres located on the bond paths represent the positions of the bond critical points.

bond paths have been argued to serve as "key-lock" mainstays in the structuralization of the molecules in inorganic molecular crystals. $^{20}$

Of the inorganic molecular materials cited above, arsenolite is possibly one of the best examples for illustrating the role played by directed bond paths in the structuralization of the molecules in a crystal. ${ }^{22}$ A perspective drawing of the structure (Figure 3) shows that its tetrahedral $\mathrm{As}_{4} \mathrm{~S}_{6}$ molecules are arranged like the $\mathrm{C}$ atoms in diamond, ${ }^{25}$ establishing that the molecules occupy only one-third of the space that would be occupied by a close-packed array of molecules. Intermolecular $\mathrm{vdW} \mathrm{As}-\mathrm{O}$ bond paths radiate from each of the four equivalent tetrahedral faces of a centrally located molecule to the tetrahedral faces of four adjacent equivalent molecules (Figure 4). ${ }^{22}$ In effect, each tetrahedral molecule is coordinated by four equivalent molecules disposed at the corners of a tetrahedron, resulting in an open tetrahedral array of equivalent molecules. Clearly, the molecules in arsenolite are not close packed in a cheek-byjowl fashion as may be anticipated if the $\mathrm{vdW}$ forces were nondirectional. ${ }^{26}$ Instead, the four faces of each tetrahedral molecule are juxtaposed with the faces of four adjacent molecules, forming an open tetrahedral array of molecules that

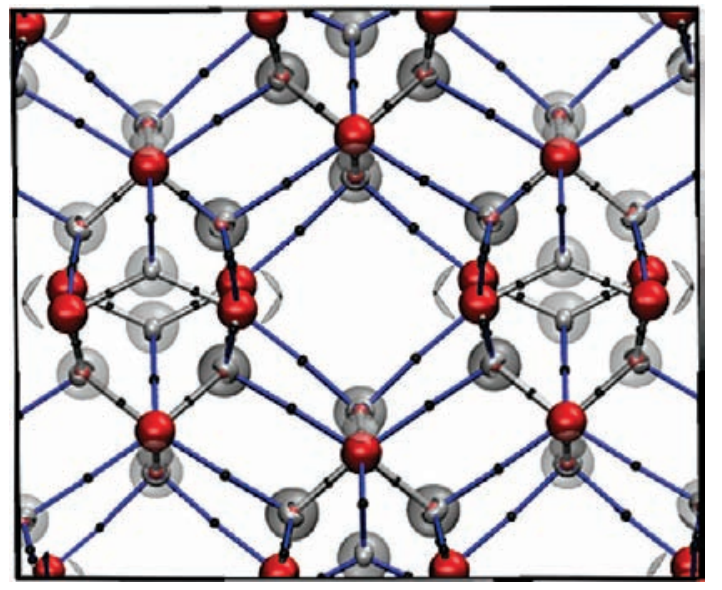

Figure 5. A mapping of Laplacian, $L(\mathbf{r})=-\nabla^{2} \rho(\mathbf{r})$, envelopes for the arsenolite structure viewed down [110]. The blue lines represent intermolecular As- $\mathrm{O}$ bond path that connects a red spherical envelope that encloses a Lewis acid domain on the As atoms and the silver earmuff-shaped envelope that caps a Lewis base domain on the $\mathrm{O}$ atoms. The paths represent as Lewis acid-base type bonded interactions.

are structuralized by directed $\mathrm{As}-\mathrm{O}$ bond paths. The end product is a face-centered cubic crystal structure with the same space group type $\mathrm{Fd} 3 \mathrm{~m}$ and octahedral vitreous colorless crystals as observed for diamond.

Figure 5 displays a composite Laplacian $L(\mathbf{r})$ envelope map superimposed on the arsenolite structure viewed down [110]. Each As atom is enclosed by a spherical envelope, $L(\mathbf{r})=-22$ $\mathrm{au}$, that represents a domain of charge depletion (a Lewis acid domain) with a skull-cap-shaped envelope of weakly concentrated ED, located at the apex of each As atom, $L(\mathbf{r})=+0.01$ au, forming a so-called $\Psi-\mathrm{AsO}_{3}$ tetrahedron. In contrast, each $\mathrm{O}$ atom is encircled in part by a ear-muff-shaped Lewis base domain envelope located on the reflex side of each As-O-As angle with a much larger positive $L(\mathbf{r})$ value, +12 au. Intermolecular $\mathrm{As}-\mathrm{O}$ bond paths branch from the spherical Lewis acid domains that encircling each As atom to the earmuff-shaped Lewis base domains on the $\mathrm{O}$ atoms of the adjacent molecules, qualifying as a Lewis acid-base directed bond path interactions. The As-O Lewis acid-base directed bond paths may be considered to serve as "key-lock" Fischer mainstays, ${ }^{27}$ providing a basis for understanding the open tetrahedral structure adopted by arsenolite. The open and highly symmetric tetrahedral structure together with the directed Lewis acid-base type interaction $\mathrm{vdW}$ bond paths that structuralize the tetrahedral molecules is compelling evidence that the vdW bond paths are not only highly directed but that the vdW forces are evidently encrypted in the Laplacian distribution of the valence electrons of the constituent atoms of the molecules. ${ }^{20}$

In addition, the low frequency Raman compressibility rigidlayer modes reported by Zallen and Slade $^{28}$ for orpiment provide credible experimental evidence that the layers of the structure are linked by well-defined directed bonded interactions. In the analysis of the rigid-layer modes, it was concluded, as observed above, that the layers are linked $\mathrm{S}-\mathrm{S}$ bonded interactions (Figure 2). Although the crystal structure and the Raman and infrared work are in accord with their assumption, our calculations are intended to provide a basis for establishing whether the layers are linked exclusively by $\mathrm{S}-\mathrm{S}$ bonded interactions. In actual fact, the calculations may also provide evidence, as observed for claudetite, $\mathrm{As}_{2} \mathrm{O}_{3},{ }^{22}$ for As-S and As-As bonded interactions between the layers and possibly for $\mathrm{S}-\mathrm{S}$ and As $-\mathrm{S}$ vdW bonded interactions within the layers. 
The goal of our study is two-fold: (1) to undertake bond path calculations to determine whether or not directed $\mathrm{S}-\mathrm{S}$ bonded interactions dominate the coupling between the layers in orpiment and (2) and to determine whether vdW bond paths other than $\mathrm{S}-\mathrm{S}$ bond paths exist between the layers and whether bond paths exist within the individual layers in addition to the shared intramolecular As $-\mathrm{S}$ bonded interactions.

Bond Paths and the Local Energy Density Properties. In a search for the bond paths representing intramolecular and intermolecular vdW bonded interactions for orpiment, the ED distribution was calculated and the bond paths were tracked for the structure, using the software packages CRYSTAL9 ${ }^{29}$ and TOPOND. ${ }^{30}$ The cell dimensions and the atomic coordinates (Table 1) are defined for orpiment by Mullen and Nowacki, ${ }^{6}$ in order to conform with the standard setting set forth in The International Tables of Crystallography. The 6-31d51G basis set for the arsenic atom ${ }^{31}$ and the $86-311 G^{*}$ basis set for the sulfur atom ${ }^{32}$ were especially optimized for these calculations. The bond critical point, $\mathbf{r}_{\mathrm{c}}$, for each bonded interaction (the point along the bond path where the gradient of the ED is zero), was found using an automated eigenvector-following searchalgorithm. The search was performed on a region of space within a radius of $6 \AA$ in diameter centered at each nonequivalent atom. Bond paths were found to exist not only for both the intramolecular $\mathrm{As}-\mathrm{S}$ bonded interactions but also for structuralizing intermolecular vdW As $-\mathrm{S}$, As-As, and $\mathrm{S}-\mathrm{S}$ interactions that organize the layers into a three-dimensional periodic array. In each case, the paths were traced by mapping the gradient vector field of the ED distribution starting at $\mathbf{r}_{\mathrm{c}}$ and terminating at the nuclei of the pair of atoms connected by the bond path. The lengths of the bond paths and the coordinates of the bond critical points for the bonded interactions calculated for orpiment are also given in Table 1. $P(\mathrm{M}-\mathrm{X})$ denotes the lengths of the bond paths connecting the atoms $\mathrm{M}$ and $\mathrm{X}$, and identify the As-S, As - As and $S-S$ bonded interactions.

As a bond path is intrinsically connected with a line of maximally negative potential energy density, Bader $^{16}$ has asserted that the presence of a bond path, together with its topologically equivalent virial path, serves as an universal indicator of a bonded interaction. Moreover, as the ED is topologically determined by the virial field, the presence of the virial path results in a line of maximum ED, a bond path and a state of electrostatic equilibrium between the pair. When these conditions are satisfied, the presence of a bond path linking a pair of atoms is believed to suffice as evidence that the pair is bonded. Indeed, all atomic interactions that result in bond paths, including vdW intermolecular interactions, are believed to be responsible for the bonded interactions in a material. ${ }^{16}$ With few exceptions, the greater the value of the ED at $\mathbf{r}_{\mathrm{c}}$, denoted $\rho\left(\mathbf{r}_{\mathrm{c}}\right)$, the stronger the bonded interaction and the shorter the equilibrium bonded distance.

Table 2 presents our results for the bond properties in orpiment. $R(\mathrm{M}-\mathrm{X})$ denotes the bond lengths connecting the atoms $\mathrm{M}$ and $\mathrm{X}$. The values of the ED at the bond critical point, $\rho\left(\mathbf{r}_{\mathrm{c}}\right)$, are given for each bonded interaction together with the values of the Laplacian, $\nabla^{2} \rho\left(\mathbf{r}_{\mathrm{c}}\right)$, both at $\mathbf{r}_{\mathrm{c}}$. The local potential energy density $V\left(\mathbf{r}_{\mathrm{c}}\right)$ (au) and the local kinetic energy density, $G\left(\mathbf{r}_{\mathrm{c}}\right)(\mathrm{au})$, both stated in atomic units, are also given in Table 2. The first block of the data in the Table presents the bond critical point properties, bcp, for the intramolecular $\mathrm{As}-\mathrm{S}$ bonded interactions; the second presents the properties for the As $-S$ vdW bonded interactions; the third represents the properties for the $\mathrm{S}-\mathrm{S}$ vdW bonded interactions; and the last represents the properties for the As-As vdW bonded interac-
TABLE 2: Bond Critical Point Properties for Orpiment Layer

\begin{tabular}{|c|c|c|c|c|}
\hline$R(\mathrm{M}-\mathrm{X})(\AA)$ & $\rho\left(\mathbf{r}_{\mathrm{c}}\right)\left(\mathrm{e} \AA^{-3}\right)$ & $\nabla^{2} \rho\left(\mathbf{r}_{\mathrm{c}}\right)\left(\mathrm{e} \AA^{-5}\right)$ & $G(\mathrm{au})$ & $V(\mathrm{au})$ \\
\hline \multicolumn{5}{|c|}{$\mathrm{As}-\mathrm{S}$} \\
\hline 2.27 & 0.599 & 1.348 & 0.0428 & -0.0715 \\
\hline 2.29 & 0.585 & 1.350 & 0.0416 & -0.0691 \\
\hline 2.29 & 0.575 & 1.409 & 0.0408 & -0.0670 \\
\hline 2.31 & 0.567 & 1.311 & 0.0395 & -0.0653 \\
\hline 2.29 & 0.571 & 1.537 & 0.0417 & -0.0674 \\
\hline 2.24 & 0.630 & 1.235 & 0.0449 & -0.0770 \\
\hline \multicolumn{5}{|c|}{ As-S van der Waals } \\
\hline $3.48^{a}$ & 0.071 & 0.621 & 0.0059 & -0.0053 \\
\hline 3.54 & 0.068 & 0.591 & 0.0056 & -0.0050 \\
\hline $3.65^{a}$ & 0.052 & 0.514 & 0.0047 & -0.0040 \\
\hline 3.22 & 0.125 & 0.933 & 0.0101 & -0.0105 \\
\hline 3.29 & 0.113 & 0.850 & 0.0091 & -0.0093 \\
\hline 3.52 & 0.071 & 0.617 & 0.0059 & -0.0053 \\
\hline $3.67^{a}$ & 0.057 & 0.482 & 0.0045 & -0.0040 \\
\hline \multicolumn{5}{|c|}{$\mathrm{S}-\mathrm{S}$ van der Waals } \\
\hline $3.24^{a}$ & 0.097 & 0.951 & 0.0092 & -0.0086 \\
\hline 3.53 & 0.070 & 0.626 & 0.0061 & -0.0056 \\
\hline $3.57^{a}$ & 0.057 & 0.602 & 0.0054 & -0.0046 \\
\hline $3.65^{a}$ & 0.058 & 0.535 & 0.0050 & -0.0044 \\
\hline 3.64 & 0.052 & 0.514 & 0.0047 & -0.0040 \\
\hline \multicolumn{5}{|c|}{ As-As van der Waals } \\
\hline $3.64^{a}$ & 0.060 & 0.525 & 0.0046 & -0.0038 \\
\hline
\end{tabular}

${ }^{a}$ Intermolecular layer bonded interactions.

(a)

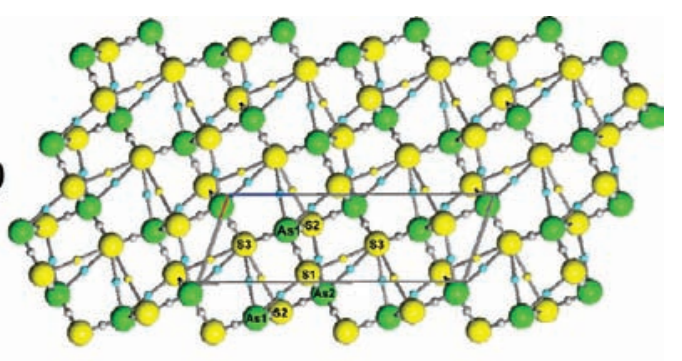

(b)

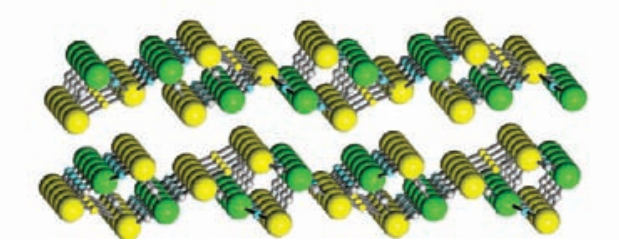

Figure 6. A drawing of the intramolecular As $-\mathrm{S}$ and $\mathrm{S}-\mathrm{S}$ bond paths that occur within the layers of orpiment. The intramolecular As $-\mathrm{S}$ bond paths are identified by silver bcps; the van der Waals As $-\mathrm{S}$ bond paths are identified by blue bcps; and the $\mathrm{S}-\mathrm{S}$ bond paths are identified by yellow bcps. Note the As1 and As2 are each connected by As $-\mathrm{S}$ bond paths to five sulfur atoms within the layer.

tions. $H\left(\mathbf{r}_{\mathrm{c}}\right)=G\left(\mathbf{r}_{\mathrm{c}}\right)+V\left(\mathbf{r}_{\mathrm{c}}\right)$ has been used to characterize a bonded interaction. ${ }^{33} \mathrm{~A}$ bonded interaction is asserted to be shared when $H\left(\mathbf{r}_{\mathrm{c}}\right)$ is negative and closed-shell when $H\left(\mathbf{r}_{\mathrm{c}}\right)$ is positive.

Intramolecular and Intermolecular Bonded Interactions. The bond critical points and the intramolecular bond paths calculated for the As $-\mathrm{S}$ bonded interactions within the strongly bonded layer of orpiment are viewed down $\mathbf{b}^{*}$ in Figure $6 \mathrm{a}$ and along $\sim \mathbf{a}$ in Figure $6 \mathrm{~b}$ for the two layers. The bond paths are represented by the silver-colored lines that connect the arsenic and sulfur atoms, and the individual bond critical points are represented by small silver-colored spheres, located about half way between the arsenic and sulfur atoms. In actual fact, these points are located, on average, $1.10 \AA$ from the arsenic 


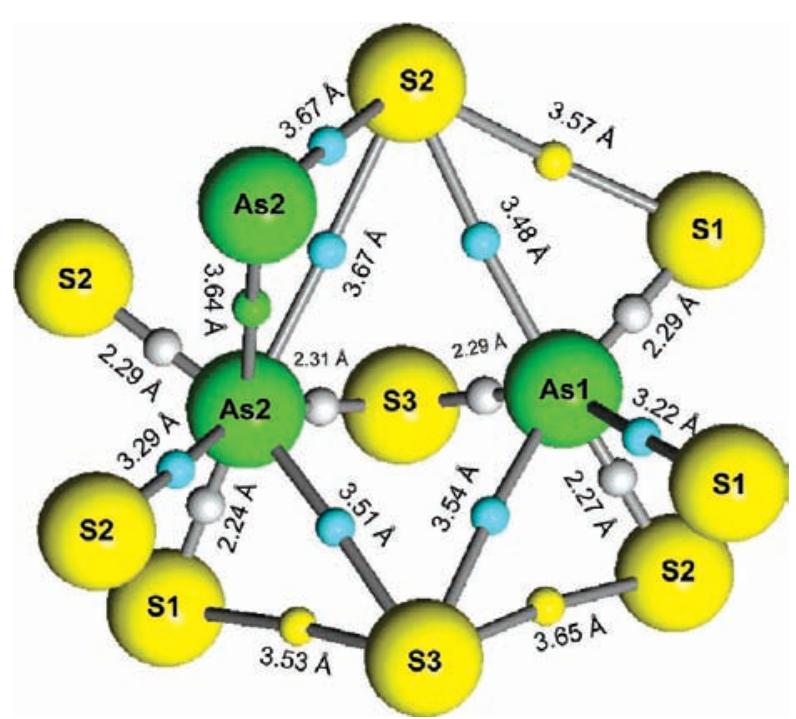

Figure 7. Drawing of the bond paths that radiate from As1 and As2 atoms in orpiment. Three intramolecular As $-\mathrm{S}$ bond paths (identified by silver bcps) and three intermolecular bond paths (identified by blue bcps) radiate from both atoms. In addition, an As2-As2 intermolecular bond path connects arsenic atoms in adjacent layers. As1 and As2 both form paths with an S2 in an adjacent layer. The sulfur atoms form a highly distorted octahedral array of sulfur atoms about each arsenic atom which together form two highly distorted $\mathrm{cp}$ monolayer domains that are roughly perpendicular to $\mathbf{a}$.

atoms and $1.19 \AA$ from the sulfur atoms along the bond paths, demonstrating that the bonded radius of the arsenic atom, $r_{\mathrm{b}}(\mathrm{As})$ $=1.10 \AA$, is $0.09 \AA$ smaller, on average, than that of the $\mathrm{S}$ atom, $r_{\mathrm{b}}(\mathrm{S})=1.19 \AA$ A . Further, the bonded radii of both atoms increase linearly $\sim 0.03 \AA$ as the $\mathrm{As}-\mathrm{S}$ bond path lengths increase from 2.24 to $2.31 \AA$, an indication that the bonded radii of both atoms increase at about the same rate as the $\mathrm{As}-\mathrm{S}$ bond path lengths.

In addition to the As $-\mathrm{S}$ intramolecular bond paths, there are three types of vdW bond paths in orpiment: As $-\mathrm{S}$ and $\mathrm{S}-\mathrm{S}$ intramolecular paths that link atoms within the layers and intermolecular As-S, S-S, and As-As paths that link atoms between the layers. The vdW intramolecular As $-\mathrm{S}$ and $\mathrm{S}-\mathrm{S}$ paths within the layer are also displayed in Figure 6, panels a and $b$. There are two nonequivalent sets of vdW intramolecular bond paths, the ones that connect the arsenic and sulfur atoms, represented by silver lines "centered" with small blue bcp spheres, and the ones that connect two sulfur atoms represented by a silver line with small yellow bcp spheres. The two nonequivalent arsenic atoms are denoted As1 and As2. Six bond paths radiate from each As1 to five sulfur atoms in the same layer and to one in an adjacent layer: seven radiate from each As2 to five sulfur atoms in the same layer and to a sulfur and an arsenic atom in an adjacent layer. The resulting face sharing $\mathrm{As}_{1} \mathrm{~S}_{6}$ and $\mathrm{As} 2 \mathrm{~S}_{6} \mathrm{As} 2$ coordination polyhedra are displayed in Figure 7 viewed down $\sim \mathbf{a}$. Both arsenic atoms are coordinated by six sulfur atoms that form highly distorted $\mathrm{AsS}_{6}$ octahedra, resulting in two domains of highly distorted close packed monolayers of sulfur atoms oriented perpendicular to $\sim \mathbf{a}$. The intramolecular As1-S and As2-S bond lengths for the two nonequivalent $\mathrm{AsS}_{3}$ coordination polyhedra measure 2.27, 2.29 $\AA(2 \times)$ and $2.24,2.29,2.31 \AA$, respectively. The additional vdW intramolecular As1-S and As2-S bond lengths measure 3.22, $3.48,3.54 \AA$ and $3.29,3.51 \AA$, respectively. The vdW intermolecular bond paths $\mathrm{S} 1-\mathrm{S} 2$, As1-S2, As2-S2, and As2-As2 that connect atoms on adjacent layers measure 3.58, $3.48,3.67$, and $3.64 \AA$, respectively. The fact that the geometric

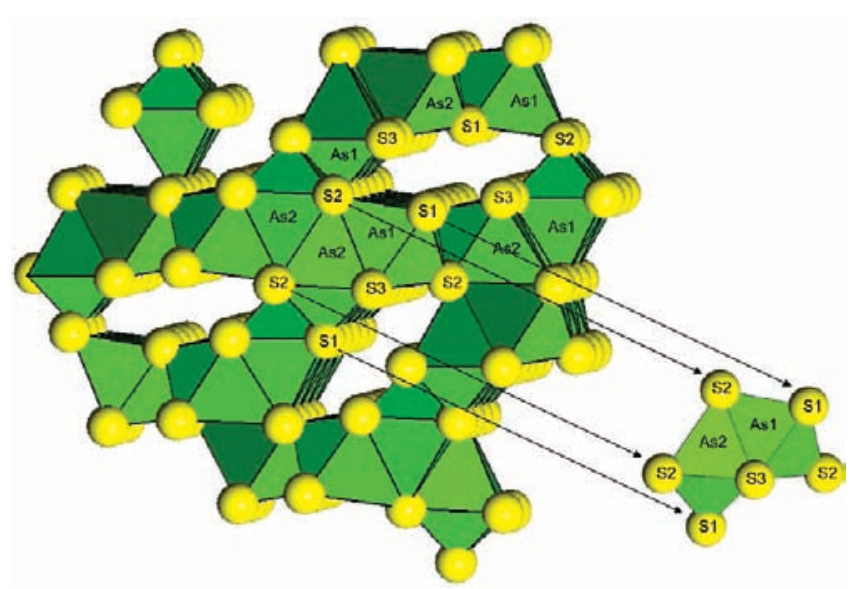

Figure 8. A drawing of the $\mathrm{AsS}_{6}$ coordinated polyhedra for orpiment viewed roughly down a. The face sharing As1S6 and As2S6 octahedra are cut from the structure by directed line segments. The channels that run along a are lined by sulfur atoms. The $S-S$ and $A s-S$ bond paths that cross the channels and form the $\mathrm{S}-\mathrm{S}$ and $\mathrm{As}-\mathrm{S}$ vdW bonded interactions are not displayed.

bond lengths are in close agreement with the bond path lengths indicates that the bond paths are highly linear, circular in cross section, and closely follow the straight line that connects the nuclei of the bonded atoms. Figure 8 is a drawing of a representative block of the orpiment structure displaying the $\mathrm{As} 1 \mathrm{O}_{6}$ and $\mathrm{As}_{2} \mathrm{O}_{6}$ coordination polyhedra viewed down $\sim \mathbf{a}$. The two polyhedra share faces and edges in the structure with the sulfur atoms forming domains of close-packed monolayers. Note that sulfur atoms line the "open" channels that run parallel to $\mathbf{a}$, a feature examined below.

There are two nonequivalent vdW S-S intramolecular bond paths within the layer that involve the three nonequivalent sulfur atoms: S1, S2, and S3. The intramolecular vdW bond paths $\mathrm{S} 1-\mathrm{S} 3$ and $\mathrm{S} 2-\mathrm{S} 3$ measure 3.52 and $3.64 \AA$, respectively, forming an $\mathrm{S} 1-\mathrm{S} 3-\mathrm{S} 2$ angle of $91.1^{\circ} . \mathrm{S} 1$ and $\mathrm{S} 2$ are both coordinated by four sulfur and three arsenic atoms whereas S3 is coordinated by two sulfur and four arsenic atoms. Of the four sulfur atoms bonded to S1, three are bonded to sulfur atoms in the same layer as $\mathrm{S} 1$, and one is bonded to a sulfur atom in an adjacent layer. Of the three arsenic atoms bonded to S1, all are in the same layer as S1. Of the four sulfur atoms bonded to S2, two are in the same layer as S2 and two are in an adjacent layer. Of the three arsenic atoms bonded to S2, all three are in the same layer as $\mathrm{S} 2$. Of the two sulfur atoms and the four arsenic atoms bonded to S3, each is in the same layer as S3. It is notable that the sulfur atoms in orpiment are linked in strings of $\mathrm{S}-\mathrm{S}$ bonded interactions that extend indefinitely throughout the structure parallel to a (Figure 9). In the string, each S1 atom is bonded to four S atoms ( $\mathrm{S} 1, \mathrm{~S} 2$, and $\mathrm{S} 3(2 \times)$ ); each $\mathrm{S} 2$ atom is bonded to two sulfur atoms (S1, S2) and each S3 atom is bonded to three sulfur atoms (S1 $(2 \times)$, S2). The S2 atoms comprise one layer together with half of the $\mathrm{S} 1$ and $\mathrm{S} 3$ atoms while the remaining half of the S1 and S3 atoms comprise the adjacent layer. The string of sulfur atoms that line the channels are displayed in Figure 8.

The intramolecular bond paths that connect the atoms within the layers are displayed in Figure 10 together with the intermolecular bond paths that connect the atoms between adjacent layers, again viewed down $\sim \mathbf{a}$. There are two nonequivalent As $-\mathrm{S}$, three nonequivalent $\mathrm{S}-\mathrm{S}$, and one nonequivalent $\mathrm{As}-\mathrm{As}$ bond paths that connect the atoms on adjacent layers. The lengths of two As-S paths are 3.65 and $3.54 \AA$; the lengths of 


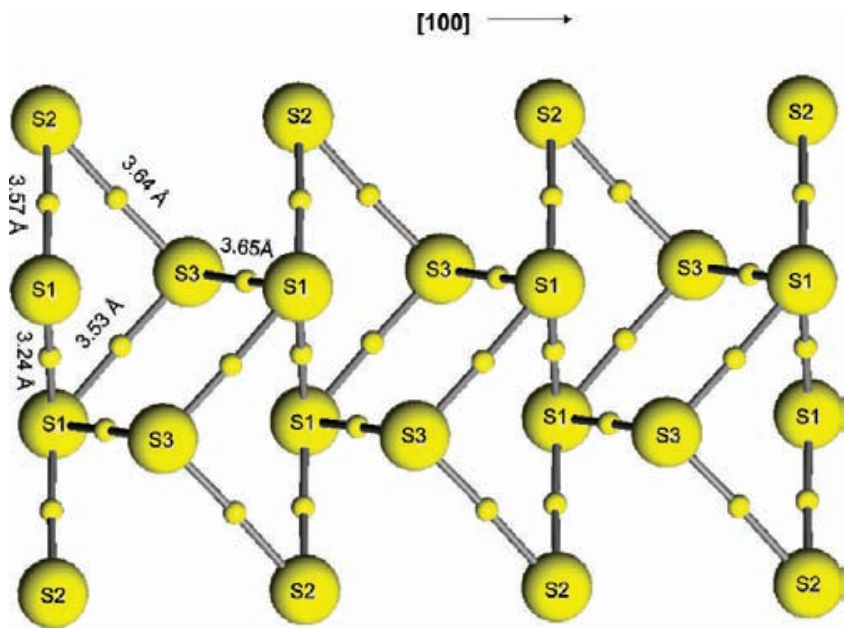

Figure 9. A representative string of sulfur atoms connected by $\mathrm{S}-\mathrm{S}$ bond paths that run parallel to [100] viewed down $\mathbf{b}^{*}$.

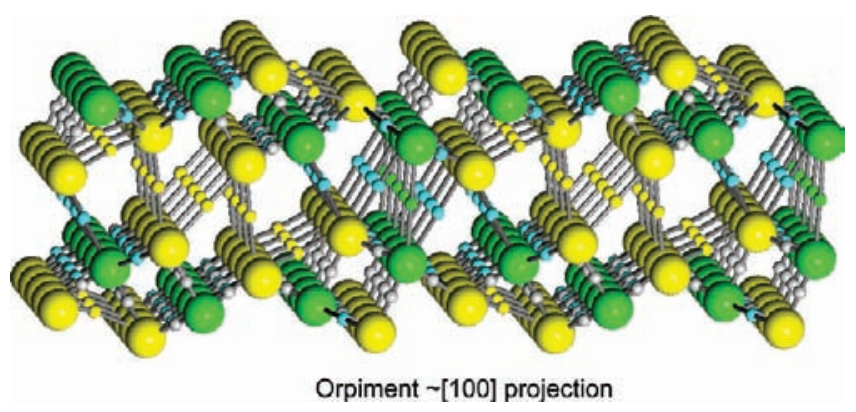

Figure 10. A drawing of the bond paths displayed in Figure 2a with the interlayer intermolecular As-S (with blue bcps), $\mathrm{S}-\mathrm{S}$ (with yellow bcps), and As-As (with green bcps) added to the figure.

the three $\mathrm{S}-\mathrm{S}$ paths are $3.24,3.57$, and $3.65 \AA$; and the length of the As-As path is $3.64 \AA$. The intermolecular vdW S-S bond paths between the layers are consistent with the layer-tolayer bonded interactions treated by Zallen and Slade. ${ }^{28}$ These intermolecular bond paths together with their substantial Ramanderived layer-layer force constants, strongly supports Slater's view that $\mathrm{vdW}$ bonded interactions behave as weak directed covalent binded interactions. The six nonequivalent $\mathrm{As}-\mathrm{S}$ bond paths range in length between 3.22 and $3.67 \AA$ with an average value of $3.39 \AA, 0.026 \AA$ smaller than the sum of the corresponding vdW radii, $3.65 \AA$, as may be expected for a bonded interaction. ${ }^{34}$ The three nonequivalent intermolecular $\mathrm{S}-\mathrm{S}$ bond paths range in value between 3.24 and $3.65 \AA$ with an average value of $3.49 \AA, 0.11 \AA$ smaller than the sum of the $\mathrm{vdW}$ radii for S. Also, the As-As bond path, $3.65 \AA$, is $0.05 \AA$ is shorter than the sum of the vdw radii. On average, all three path lengths are shorter than their vdW radii sum values, a result that indicates that the three types of $\mathrm{vdW}$ bonded interactions play a nontrivial role in stabilizing the structure. An examination of Figure 10 shows that an equal number of intermolecular $S-S$ and As $-\mathrm{S}$ bond paths with a single As-As occur in between the layers. Collectively, these bond paths indicate that the splittings of the Raman spectra may be ascribed to a combination of $\mathrm{S}-\mathrm{S}, \mathrm{As}-\mathrm{S}$, and As-As bonded interactions rather than $\mathrm{S}-\mathrm{S}$ bonded interactions exclusively.

The $\rho\left(\mathbf{r}_{\mathrm{c}}\right)$ and $R(\mathrm{~S}-\mathrm{S})$ data calculated for orpiment (plotted as red triangles) are compared in Figure 11 with those for native sulfur (blue spheres) and for several thioarsenide molecular crystals (crosses). The experimental intermolecular $\mathrm{S}-\mathrm{S}$ bond lengths for orpiment, orthorhombic sulfur and the thioarsenide crystals decrease in a nonlinear fashion with the increasing value

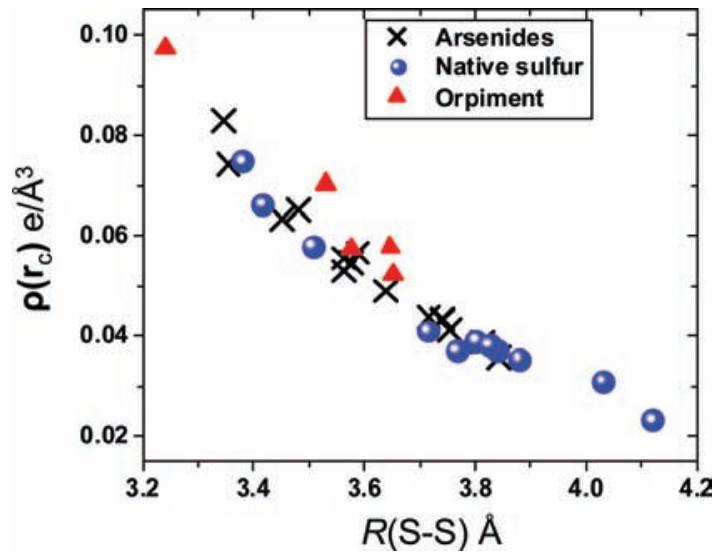

Figure 11. The electron density, $\rho\left(\mathbf{r}_{c}\right)$, determined at the bond critical points, $\mathbf{r}_{\mathrm{c}}$, plotted with respect to the experimental bond lengths, $R(\mathrm{~S}-\mathrm{S})$, for orpiment (solid red triangles), native sulfur (blue spheres), and for the arsenide molecular crystals (crosses) realgar (AsS), $\beta$-AsS, pararealgar (AsS), uzonite $\left(\mathrm{As}_{4} \mathrm{~S}_{3}\right)$, alacranite $\left(\mathrm{As}_{8} \mathrm{~S}_{9}\right)$, and $\alpha$ - and $\beta$-dimorphite $\left(\mathrm{As}_{4} \mathrm{~S}_{5}\right)$. The intermolecular vdW S-S bond lengths in orpiment measure $3.24,3.57$, and $3.65 \AA$, and the intramolecular vdW bond lengths measure 3.53 and $3.64 \AA$.

of $\rho\left(\mathbf{r}_{\mathrm{c}}\right)$, the larger the value of $\rho\left(\mathbf{r}_{\mathrm{c}}\right)$, the shorter the bond length and its associated bond path. The commingling of the data sets indicates that a close correspondence exists among the $\mathrm{S}-\mathrm{S}$ interactions occurring among the molecules in orthorhombic sulfur, the thioarsenides, and orpiment. The correspondence indicates that the $\mathrm{S}-\mathrm{S}$ bonded interactions in sulfur and the thioarsenide crystals represent directed $\mathrm{S}-\mathrm{S}$ bonded interactions, as we have shown for orpiment. It is notable that the shortest $\mathrm{S}-\mathrm{S}$ bond, $R(\mathrm{~S}-\mathrm{S})=3.24 \AA$, occurs between the layers of orpiment, associated with the largest $\rho\left(\mathbf{r}_{\mathrm{c}}\right)$ value of $\sim 0.10 \mathrm{e} / \AA^{3}$.

The value of $\nabla^{2} \rho\left(\mathbf{r}_{\mathrm{c}}\right)$ for the $\mathrm{S}-\mathrm{S}$ intermolecular interactions for orpiment, sulfur, and the arsenides increases linearly with decreasing bond length. However, it is independent of the bond lengths for As $-\mathrm{S}$ intramolecular bonded interactions. Further, the bond radii for the arsenic and sulfur atoms for all of the bonded interactions are highly correlated with bond length with each clustering along two separate but parallel linear trends.

The connection between the bond paths and the Laplacian, $L(\mathbf{r})=-\nabla^{2} \rho(\mathbf{r})$, isosurface envelopes of the ED distribution for orpiment are displayed in Figure 12. The intramolecular As $-\mathrm{S}$ bond paths are silver, and the intermolecular As $-\mathrm{S}, \mathrm{S}-\mathrm{S}$, and As-As bond paths are blue, yellow, and green, respectively. The topology of $L(\mathbf{r})$ for the As-As intermolecular bonded interaction are comparable with those recorded for native bulk arsenic, and the intermolecular $\mathrm{S}-\mathrm{S}$ bonded interactions are comparable with those observed for native sulfur. Each $\mathrm{S}$ is enclosed in part by a ear-muff-shaped lone pair envelope mapped at the $L(\mathbf{r})=1.25$ au level, and each As is enclosed by a spherical envelope mapped at the $L(\mathbf{r})=-7.0$ au level and capped by a very diffuse skull-cap-shaped base envelope mapped at the $L(\mathbf{r})=0.001$ au level. As observed for the thioarsenides, the As $-\mathrm{S}$ bond paths connect the Lewis base lone pair domains on the sulfur atoms with Lewis acid domains on the arsenic atoms and qualify as directed $\mathrm{As}-\mathrm{S} v \mathrm{vdW}$ bonded interactions. ${ }^{18}$

According to the Makovicky and Mumme ${ }^{36}$ "so-called micelle model", the orpiment structure is pictured as consisting of layers separated by arsenic lone pair domains ("planar micelles") crosslinked by weak vdW directed bonded interactions. ${ }^{7}$ Further, the string of sulfur atoms in orpiment that parallels [100] and is cross-linked by $\mathrm{S}-\mathrm{S}$ and $\mathrm{As}-\mathrm{S}$ bond paths may likewise be 


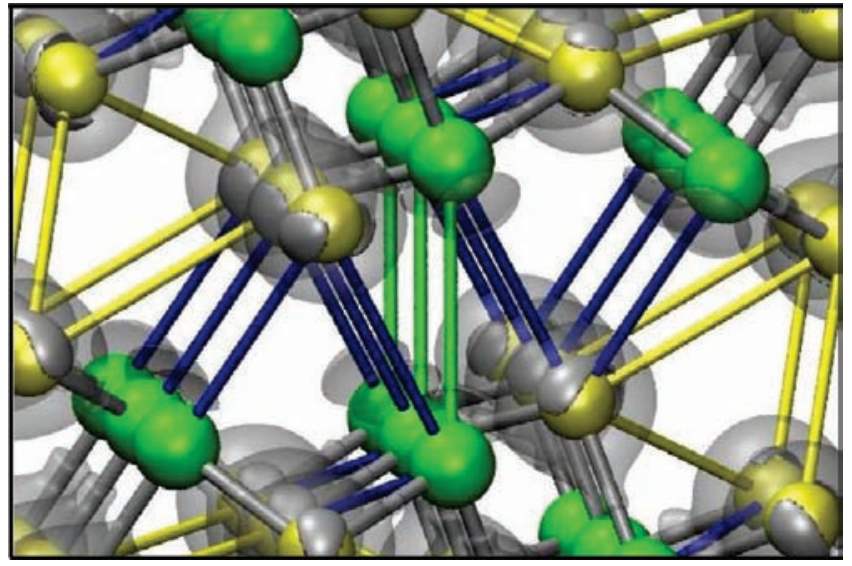

Figure 12. Envelopes of the Laplacian of the electron density, ED. distribution, $-\nabla^{2} \rho(\mathbf{r})$. Each sulfur atom (colored yellow) is partly encircled by a ear-muff-shaped envelope $(L(\mathbf{r})=1.25 \mathrm{au})$ of locally concentrated ED (colored silver) and each arsenic atom green is enclosed by green spherical envelope $(L(\mathbf{r})=-7.0 \mathrm{au})$ of locally depleted ED capped by very diffuse skull cap shaped envelope $(L(\mathbf{r})$ $=0.001 \mathrm{au}$ ) of locally concentrated ED. The intramolecular bond paths are colored silver and intermolecular $\mathrm{As}-\mathrm{S}, \mathrm{S}-\mathrm{S}$ and $\mathrm{As}-\mathrm{As}$ bond paths are colored blue, yellow, and green, respectively. The ear-muffshaped envelopes of the $\mathrm{S}$ atoms line the channels of sulfur atoms that parallel a.

considered as a "so-called lone pair micelle". However, the $L(\mathbf{r})$ maps fail to provide evidence for a system of well-developed lone electron pairs associated with the arsenic atoms. The bond paths displayed for orpiment in Figure 12 are consistent with this picture in that the layers are cross-linked by directed vdW As $-\mathrm{S}$ and $\mathrm{S}-\mathrm{S}$ interactions. The $L(\mathbf{r})$ maps indicate that lone pairs on the sulfur atoms are much better developed. Further, it is notable that they line the channels formed by the strings of sulfur atoms that parallels a as may be expected for a so-called micelle. It is our sense that a so-called micelle is little more than a domain in a structure that is cross-linked by directed $\mathrm{vdW}$ bonded interactions. On the basis of the local energy density (Table 2), $H\left(\mathbf{r}_{\mathrm{c}}\right)$, the intramolecular bonded interactions qualify as shared interactions while the vdW interactions qualify as closed shell interactions. ${ }^{33}$

\section{Concluding Remarks}

Bader $^{16}$ defined a bond path as a line of ED that connects a pair of bonded atoms along which the value of the ED is a maximum relative to any neighboring line. In a study of the forces in molecules, Feynman ${ }^{12}$ concluded that vdW forces result by dint of the accumulation of the ED between the nuclei of a bonded pair of atoms, providing raison d'etre for a bond path. As such, a bond path is an observable feature in the ED distribution that connects a pair of bonded atoms with a concomitant stabilization of the structure. ${ }^{17,37}$ It may be noted, however, that a bond path is an indicator of a bonded interaction, ${ }^{16}$ but it is not a chemical bond as stressed by Bader. ${ }^{17}$ As documented, unlike a bond path, a chemical bond is an unobservable property inasmuch as there is no known linear Hermitian operator that can be defined for a chemical bond. But just because it is an unobservable and cannot be determined experimentally does not mean that the concept has not been extraordinarily useful in our study of materials. Without a doubt, it has played a pivotal role in advancing our understanding of the structures, properties, and reactivity of materials, among other things, at the atomic level. For example, since Linus Pauling ${ }^{38}$ wrote his classic paper "The Nature of the Chemical
Bond", more than 30000 papers have been published about one or more aspects of the concept, evidence of the profound impact that the chemical bond has had on chemical thinking and research.

As a rule, vdW bonded interactions are defined to be attractive nondirectional interactions other than those ascribed to bona fide chemical intramolecular bonded interactions. For orpiment, it was expected that directed vdW bond paths would be found to exist between the layers of the structure, given the compelling spectral evidence found by Zallen and Slade $^{28}$ for substantial layer-layer vibrational restoring forces for the interlayer bonded interactions. The spectral evidence along with specific layer-layer directed bond paths identified in this report, provide support for Slater's belief "that there is no very fundamental distinction between van der Waals binding and covalent binding". The directed bond paths are also consistent with Kitaigorodskii's ${ }^{8}$ conclusion that the structuralizing forces in inorganic molecular systems are largely directional. The evidence is also consistent with his observation that the same type of behavior obtains for the interactions, when considered in terms of the ED distribution, which is ultimately responsible for the interatomic forces that link a pair atoms. It also provides support for Bader's ${ }^{17}$ assertion that van der Waals interactions are not distinct from other bonded interactions as customarily assumed in the definition of a vdW interaction. ${ }^{39}$ The bond paths calculated for orpiment indicate that $\mathrm{S}-\mathrm{S}, \mathrm{As}-\mathrm{S}$ and $\mathrm{As}-\mathrm{As} \mathrm{vdW}$ bonded interactions exist between the layers and that $A s-S$ and $S-S$ vdW interactions exist within the layers. The bond paths established in these studies support the general picture that the layers in orpiment are held together by the bonded interactions established in the Raman study, ${ }^{28}$ but it is not clear from an examination of the bond paths which of the three bonded interactions give rise to the rigid layer interlayer modes. However, given the greater number of interlayer $S-S$ and $A s-S$ bond paths, it would appear that the layers are not only linked by $S-S$ bonded interactions but also by As $-\mathrm{S}$ bonded interactions with As-As interactions playing a minor role. Further, as emphasized in this report, the open and highly symmetric tetrahedral structures of both arsenolite and sénarmontite ${ }^{21}$ together with the directed Lewis acid-base type interaction vdW bond paths that structuralize the molecules is compelling evidence, that the As-O and $\mathrm{Sb}-\mathrm{O}$ vdW bond paths are not only highly directed but that the vdW forces are evidently encrypted in the Laplacian distribution of the valence electrons of the arsenic, antimony, and oxygen atoms of the molecules.

Acknowledgment. The National Science Foundation and the U.S. Department of Energy are thanked for supporting this study with Grants EaR-0609885 (N.L.R. and G.V.G.), EAR-0609906 (R.T.D.), and DE-FG02-97ER14751 (D.F.C.). K.M.R. acknowledges a grant from the U.S. Department of Energy (DOE), Office of Basic Energy Sciences, Geoscience Division, and computational facilities and support from the Environmental Molecular Sciences Laboratory (EMSL) at the Pacific Northwest National Laboratory (PNNL). The computations were performed in part at the EMSL at PNNL. The EMSL is a national scientific user facility sponsored by the U.S. DOE Office of Biological and Environmental Research. PNNL is operated by Battelle for the DOE under contract DEaC06-76RLO 1830. We are grateful to one of the reviewers for suggesting that a section be added to the manuscript for the benefit of the "nonspecialist reader" that emphasizes that bond paths are indicative of bonded interactions, not chemical bonds. This suggestion resulted in a substantial improvement in the manuscript. G.V.G. also wishes to thank Professors Richard F.W. Bader at McMaster University, 
T. Daniel Crawford at Virginia Tech, Dieter Cremer at Southern Methodist University, and Vladimir Tsirelson at Mendeleev University of Chemical Technology, Moscow, Russia for their enlightening discussions and comments about the chemical bond and bonded interactions.

\section{References and Notes}

(1) Zallen, R.; Drews, R. E.; Emerald, R. L.; Slade, M. L. Phys. Rev. Lett. 1971, 26, 1564. 3866 .

(2) Besson, J. M.; Cernogora, J.; Zallen, R. Phys. Rev. B 1980, 22,

(3) Zallen, R. High Pressure Res. 2004, 24, 117.

(4) Zallen, R.; Slade, M. L.; Ward, A. T. Phys. Rev. B 1971, 3, 4257.

(5) Morimoto, N. Mineral. J. Jpn. 1954, 1, 160.

(6) Mullen, D. J. E.; Nowacki, W. Z. Kristallogr. 1972, 136, 48.

(7) Makovicky, E. Rev. Mineral. Geochem. 2006, 61, 7.

(8) Kitaigorodskii, A. I. Organic Chemical Crystallography; Academy of Sciences of USSR: Moscow, 1955.

(9) Dunitz, J. D.; Gavezzotti, A. Chem. Soc. Rev. 2009, 38, 2622.

(10) Dunitz, J. D.; Gavezzotti, A. Angew. Chem., Int. Ed. 2005, 44, 1766.

(11) Defonzo, A. P.; Tauc, J. Phys. Rev. B 1978, 18, 6957.

(12) Feynman, R. P. Phys. Rev. 1939, 56, 340.

(13) Slater, J. C. J. Chem. Phys. 1972, 57, 2389.

(14) Bader, R. F. W. Atoms in Molecules; Oxford Science Publications: Oxford, UK, 1990.

(15) Bone, R. G. A.; Bader, R. F. W. J. Phys. Chem. 1996, 100, 10892

(16) Bader, R. F. W. J. Phys. Chem. A 1998, 102, 7314.

(17) Bader, R. F. W. J. Phys. Chem. A 2009, 113, 10391

(18) Tsirelson, V. G.; Zou, P. F.; Tang, T. H.; Bader, R. F. W. Acta Crystallogr., Sect. A 1995, 51, 143.

(19) Boese, R.; Boese, A. D.; Blaser, D.; Antipin, M. Y.; Ellern, A.; Seppelt, K. Angew. Chem., Int. Ed. Engl. 1997, 36, 1489.
(20) Scherer, W.; Spiegler, M.; Pedersen, B.; Tafipolsky, M.; Hieringer, W.; Reinhard, B.; Downs, A. J.; McGrady, G. S. Chem. Commun. 2000, 635 .

(21) Whitten, A. E.; Dittrich, B.; Spackman, M. A.; Turner, P.; Brown, T. C. Dalton Transactions 2004, 23.

(22) Gibbs, G. V. Wallace, A. F; Cox, D. F. Dove, P. M; Downs, R. T.; Ross, N. L.; Rosso, K. M. J. Phys. Chem. A 2009, 113, 736.

(23) Tsirelson, V. G.; Shishkina, A. V.; Stash, A. I.; Parsons, S. Acta Crystallogr., B 2009, 65.

(24) Bader, R. F. W.; MacDougall, P. J. J. Am. Chem. Soc. 1985, 107, 6788

(25) Bozorth, R. M. J. Am. Chem. Soc. 1923, 45, 1621.

(26) Pertlik, F. Czech. J. Phys. 1974, 28, 170.

(27) Bader, R. F. W.; Hernandez-Trujillo, J.; Cortes-Guzman, F. J. Comput. Chem. 2007, $28,4$.

(28) Zallen, R.; Slade, M. Phys. Rev. B 1974, 9, 1627.

(29) Saunders, V. R.; Dovesi, R.; Roetti, C.; Causa, M.; Harrison, N. M.; Orlando, R.; Apra, E. CRYSTAL98 User's Manual; University of Torino: Torino, Italy, 1998.

(30) Gatti, C. TOPOND96 User's Manual; CNR-CSRSRC: Milano, Italy, 1997

(31) Towler, M. D.; Dovesi, R.; Saunders, V. R. Phys. Rev. B 1995, 52,10150 .

(32) Lichanot, A.; Apra, E.; Dovesi, R. Phys. Status Solidi B 1993, 177 , 157.

(33) Cremer, D.; Kraka, E. Croat. Chem. Acta 1984, 57, 1259.

(34) Carroll, M. T.; Bader, R. F. W. Mol. Phys. 1988, 65, 695.

(35) Gibbs et al., in preparation.

(36) Makovicky, E.; Mumme, W. G. Neues Jahrb. Mineral., Abh. 1983 , 147, 58.

(37) Gatti, C. Z. Kristallogr. 2005, 220, 399

(38) Pauling, L. J. Am. Chem. Soc. 1931, 53, 3225

(39) Nic, M.; Jirat, J.; Kosata, B. IUPAC Compendium of Chemical Terminology; 2nd (The "Gold Book") ed.; McNaught, A., Wilkinson, A., Eds.; Blackwell Scientific Publications: Oxford, 1997.

JP102391A 